\title{
THE DEVELOPMENT OF ADAPTIVE HEAD MOVEMENTS FOLLOWING ENUCLEATION
}

\author{
J. J. MAROTTA ${ }^{1}$, T. S. PERROT ${ }^{2}$, D. NICOLLE ${ }^{3}$ and M. A. GOODALE ${ }^{2}$ \\ London, Ontario, Canada
}

\begin{abstract}
SUMMARY
Recent work in our laboratory has revealed that enucleated patients produce large lateral and vertical head movements during visually guided grasping. These movements may allow them to maximise the use of retinal motion cues in planning and controlling their grasp. The aim of the present study was to determine whether the tendency to produce these adaptive head movements increases as a function of time since enucleation. We tested a group of 12 enucleated patients in whom the time between surgery and testing varied from 2 weeks to 35 years (mean = 11.2 years). These patients were required to reach out and grasp oblong blocks of different sizes at different distances. Correlational tests revealed an increase in the proportion of self-generated lateral and vertical head movements versus forward head movements as a function of post-enucleation time $\left(r(s)_{(12)}=0.68\right.$, $p<0.025$ and $r(\mathrm{~s})_{(12)}=0.65, p<0.025$, respectively). This suggests that enucleated patients may be adapting to living with one eye by learning to increase the proportion of their lateral and vertical head movements during the performance of skilled motor acts.
\end{abstract}

It has been suggested that enucleated patients undergo some form of adaptation since patients who still have good vision in their remaining eye typically report no permanent visual difficulty following enucleation and return to normal activities within a year, often making the adjustment in less than 1 month. ${ }^{1,2}$ Previous research has highlighted the strength and effectiveness of the use of visual motion depth cues (generated by translational and rotational movements of the head) by enucleated patients when trying to resolve small depth differences. $^{3,4}$ A recent study. in our laboratory was

From: ${ }^{1}$ Neuroscience Programme, ${ }^{2}$ Department of Psychology and ${ }^{3}$ Department of Ophthalmology, University of Western Ontario, Canada.

Correspondence to: Jonathan Marotta, Neuroscience/Department of Psychology, Social Science Centre, University of Western Ontario, London, Ontario, Canada N6A-5C2. Fax: (519)-6613961. performed to determine whether or not enucleated patients (who were tested more than 1 year after surgery) generate more head movements than control subjects, who either wore an eye-patch or used binocular vision, during the performance of a visually guided grasping movement. ${ }^{5}$ The study clearly revealed that enucleated patients produced larger and faster resultant head movements, composed mainly of lateral and vertical movements, while control subjects under the monocular viewing condition generated larger forward head movements. This result suggested that the enucleated patients may have been exaggerating the natural head (and torso) movements that occur during a reach in order to better utilise retinal motion cues to aid in manual prehension. It is possible that these patients may have, over time, learned that horizontal head movements are the most effective form of head motion for the production of motion depth cues ${ }^{4,6,7}$ which can assist them in calibrating the distance (and size) of objects that they might wish to grasp.

This paper describes an examination of head movements produced by a group of enucleated patients, in whom the post-enucleation time varied from 2 weeks to 35 years, during the performance of a visually guided grasping movement. The aim of this study was to determine whether the adaptive head movements produced by enucleated patients change over the post-enucleation period from forward head movements, as displayed by normal subjects wearing an eye-patch, to lateral and vertical head movements, as demonstrated by the enucleated patients in our previous study.

\section{MATERIALS AND METHODS}

\section{Subjects}

As Table I shows, the subjects consisted of 12 adult, enucleated patients (10 men, 2 women; mean age 39.6 years; 5 right eye enucleations, 7 left eye enucleations; enucleation range 0.55 months to 35 years ago, mean time of enucleation 11.2 years ago) 
Table I. Summary of enucleated patients' histories

\begin{tabular}{cclc}
\hline Patient & Sex/age (yr) & \multicolumn{1}{c}{ Enucleated eye and reason for enucleation } & Post-enucleation period (months) \\
\hline 1 & F/63 & Right eye: melanoma & 0.55 \\
2 & M/66 & Left eye: cancer & 1.6 \\
3 & M/52 & Left eye: melanoma & 4.5 \\
4 & M/29 & Right eye: phthisis bulbi & 8.0 \\
5 & M/38 & Left eye: Accident & 11.0 \\
6 & M/28 & Right eye: Accident & 17.0 \\
7 & M/49 & Right eye: Malnutrition (vitamin A deficiency) & 63.0 \\
8 & M/19 & Left eye: Cornea build-up & 108.0 \\
9 & M/23 & Left eye: Coat's disease & 228.0 \\
10 & M/33 & Left eye: Blind for 28 years; surgery 5 years prior to testing & 336.0 \\
11 & F/37 & Right eye: Accident & 414.0 \\
12 & M/38 & Left eye: Accident & 420.0 \\
\hline
\end{tabular}

All subjects participated for pay, were strongly righthanded, as determined by a modified version of the Edinburgh Handedness Inventory, ${ }^{8}$ and had normal or corrected-to-normal acuity.

\section{Apparatus}

Subjects sat at a table (100 cm wide and $61 \mathrm{~cm}$ deep) with a matte grey surface. A circular, 1-cm-diameter microswitch button located $15 \mathrm{~cm}$ from the subject functioned as the start position for each reaching movement. This start button was located directly in front of the subject along the sagittal plane of the body. A circular fluorescent lamp was suspended approximately $80 \mathrm{~cm}$ above the table surface. This lamp was illuminated by the experimenter from a remote switch that also triggered the start of data collection.

Three red, oblong wooden blocks $(2 \mathrm{~cm}$ thick $)$ with surface areas of $2 \times 5 \mathrm{~cm}, 3 \times 7.5 \mathrm{~cm}$ and $5 \times 12.5 \mathrm{~cm}$ were used. The objects were positioned with their long axis perpendicular to the body midline. The underside of each of the objects contained an embedded magnet which, when placed in position, closed one of three magnetic switches located under the table surface at distances of 20, 30 or $40 \mathrm{~cm}$ from the microswitch along the subject's midline. When subjects picked up the object, contact between the two magnets was broken, signalling the end of collection for a given trial.

Three infrared-light-emitting diodes (IREDs) were mounted on a head piece that had been painted flat black and was attached to a Velcro band placed around the subject's head. The headpiece consisted of a T-bar mounted on a cork base, with an IRED angled at $45^{\circ}$ at the end of each arm of the T-bar, $10 \mathrm{~cm}$ apart. The head-piece extended two of the IREDs $7.5 \mathrm{~cm}$ from the subject's forehead. A third IRED was positioned on the cork base of the head-piece so that it extended $4 \mathrm{~cm}$ from the subject's forehead.

The IREDs were monitored by two high-resolution infrared-sensitive cameras positioned approximately $2 \mathrm{~m}$ from the subject. The positions of the IREDs were digitised at a rate of $100 \mathrm{~Hz}$ into twodimensional coordinates and then passed on to the data collection system of a WATSMART computer (Waterloo Spatial Motion Analysis and Recording Technique, manufactured by Northern Digital Inc., Waterloo, Ontario).

\section{Procedure}

At the beginning of the test session, subjects completed a consent form which clearly detailed the experimental methods and were then given the handedness questionnaire. Subjects were then seated at the testing table and instructed to pick up the target object with the thumb and index finger of their right hand across the narrow part of the block as soon as they could see it after the overhead light was illuminated. They were instructed to reach as quickly, accurately and 'naturally' as possible.

At the beginning of each trial, subjects placed the tips of the index finger and thumb of their right hand on the start button. Between trials, the room lights were extinguished and subjects were instructed to keep their eyes closed during this time. Once a block had been placed in a given position by the experimenter, subjects were given a verbal signal to open their eyes and the overhead light was turned on, which started the collection of the trial.

Subjects were administered testing blocks of 45 experimental trials, each consisting of five instances of each of the nine possible distance and object size combinations. Trial presentation was random and each testing block was preceded by a series of five practice trials. Any experimental trials in which the subject dropped an object was repeated at the end of a given block. The testing session lasted for approximately 45 minutes.

\section{Data Processing}

Stored sets of two-dimensional (2D) coordinates were converted into three-dimensional (3D) coordinates off-line and filtered (with a low pass secondorder Butterworth filter with a $7 \mathrm{~Hz}$ cut-off). The centre IRED mounted on the head-piece provided information about the head movements made by the subject during the entire reach. In addition, all three IREDs were used to calculate the amount of head 
rotation that occurred during the production of head movements.

\section{RESULTS}

Although the enucleated patients' overall (resultant) head movements did not vary as a function of postenucleation time $\left(r(\mathrm{~s})_{(12)}=-0.07, p>0.05\right)$, there was some indication that the different components of that overall movement did. As seen in Fig. 1, there was an increase in the magnitude of patients' vertical $(Y)$ head movements $\left(r(s)_{(12)}=0.68, \quad p<0.025\right)$ and corresponding decrease in their forward $(Z)$ head movements $\left(r(\mathrm{~s})_{(12)}=-0.50, p<0.05\right)$ as a function of post-enucleation time. As well, although not statistically significant, there appeared to be an increase in the amplitude of the lateral $(X)$ head movements as a function of time since enucleation $\left(r(\mathrm{~s})_{(12)}=0.29\right.$, $p>0.05)$. In order to examine the change in the proportional relationship between the directional components of the patients' head movements and, at the same time, to reduce the influence of

A

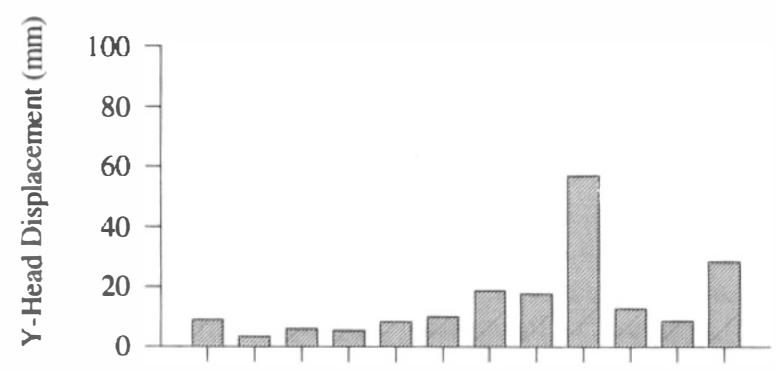

B

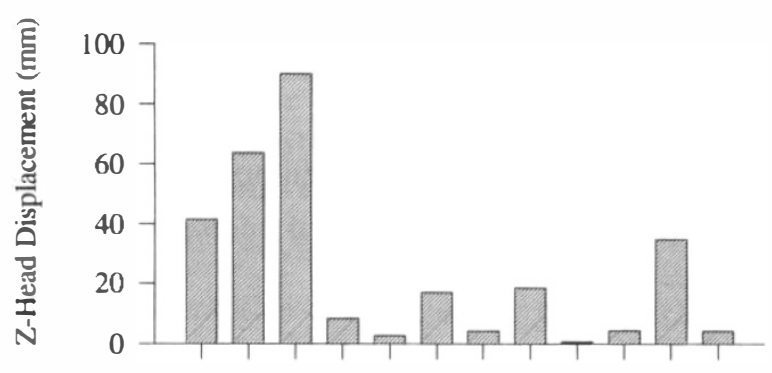

C

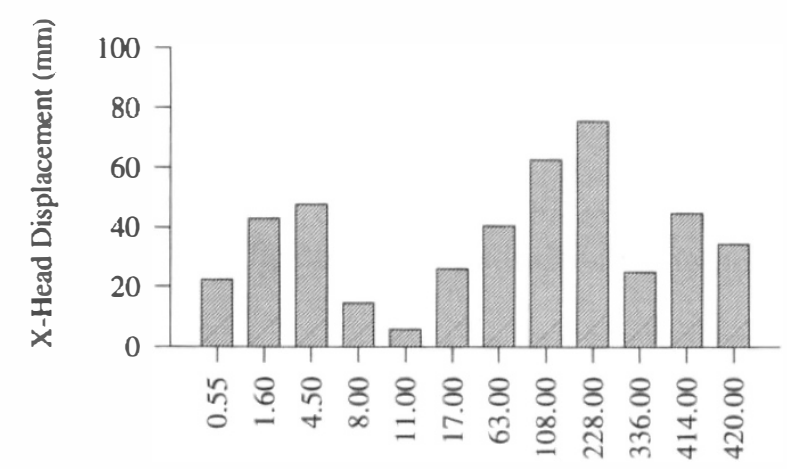

Post-Enucleation Period (months)

Fig. 1. The effects of the post-enucleation period on: (A) $Y$-head displacement. (B) Z-head displacement, and (C) Xhead displacement. individual differences in overall movement production between subjects, the ratios of the lateral and vertical head movements versus forward head movements were examined. As seen in Fig. 2, there was an increase in the ratio of lateral $(X)$ head movements versus forward $(Z)$ head movements by enucleated patients over their post-enucleation period $\left(r(\mathrm{~s})_{(12)}=0.66, p<0.025\right)$. In addition, there was an increase in the ratio of vertical $(Y)$ head movements versus forward $(Z)$ head movements over post-enucleation time $\left(r(\mathrm{~s})_{(12)}=0.65, p<0.025\right)$.

Although the correlation coefficient was not statistically significant, there appeared to be a slight increase in the amount of head rotation produced by enucleated patients as a function of time since enucleation. Because the previous literature suggests that most enucleated patients return to normal activities within 1 year, ${ }^{5}$ we separated the patients into groups of 'recent' (tested less than 1 year after enucleation) and 'late' (tested more than 1 year after enucleation) enucleated patients. When we examined these groups we found that the mean head rotation produced by recently enucleated patients in the horizontal plane $\left(2.32^{\circ} ; \mathrm{SD}=1.95^{\circ}\right)$ was found to be significantly less than the amount of head rotation
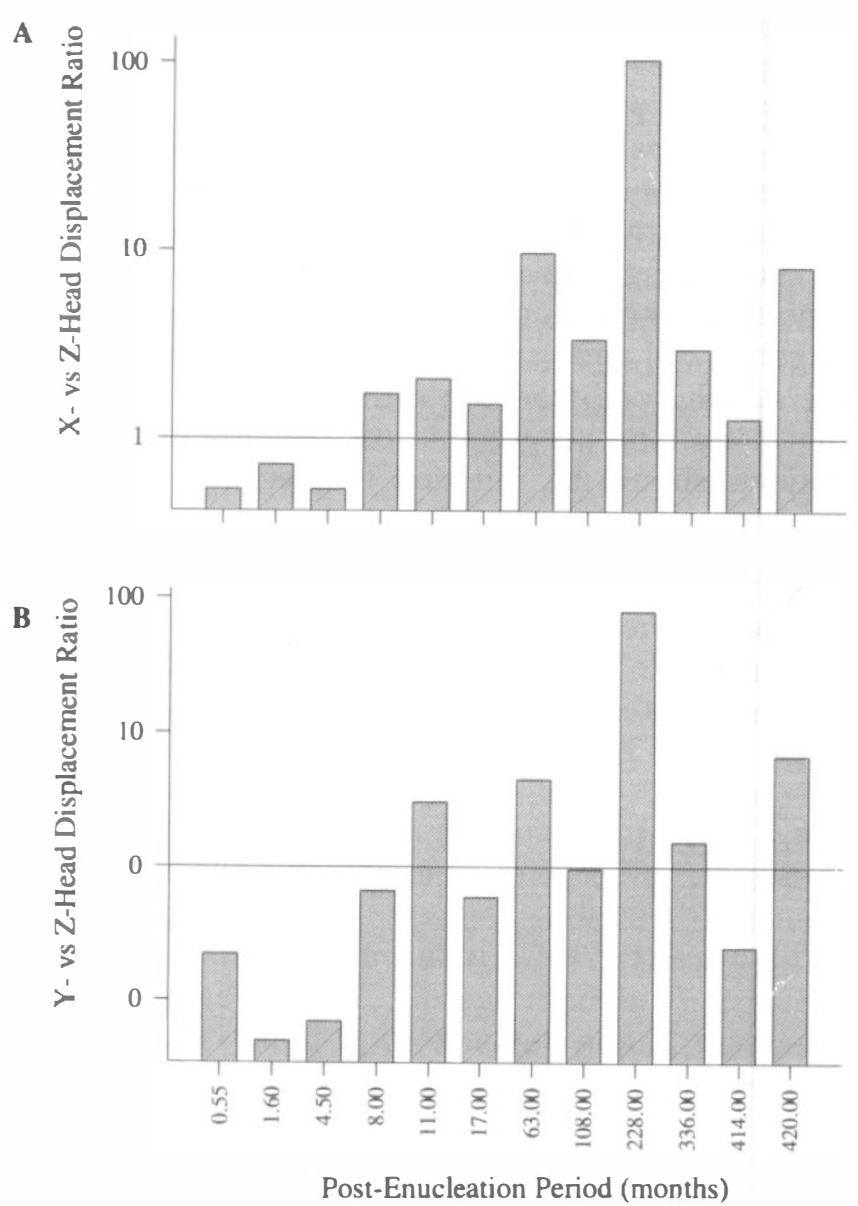

Fig. 2. The effects of the post-enucleation period on: (A) the ratio of $X$-versus $\mathrm{Z}$-head displacement, and (B) the ratio of $Y$-versus Z-head displacement. 
made by late enucleated patients (tested $>1$ year) $\left(3.24^{\circ} ; \mathrm{SD}=2.21^{\circ}\right)\left(t_{(106)}=2.44, \quad p<0: 01\right)$. Nevertheless, despite the fact that the amount of rotation increased as a function of post-enucleation time, the amplitude of that rotation, even in the late enucleated patients, was typically less than $7^{\circ}$.

\section{DISCUSSION}

Although recently enucleated patients produce head movements that are just as large as those produced by late enucleated patients, it appears that over time patients change the way in which they make those head movements. Enucleated patients seem to be adapting to living with one eye by learning to generate proportionally larger lateral $(X)$ and vertical $(Y)$ head movements, as opposed to the forward $(Z)$ head movements typically produced by recently enucleated patients and normal subjects reaching under monocular viewing conditions. While forward head movements can generate 'looming' motion cues which are potentially useful as a source of information of egocentric distance and 'time-tocontact', any positional or velocity information generated by the changing retinal image would be less than half as large, and thus possibly not as helpful; as that generated by comparable displacements in the lateral or vertical plane. ${ }^{5,6}$

While the recently enucleated patients produce fewer horizontal head rotations than the late enucleated patients, the amount of rotation was still very small and thus contributed little to the lateral movements that were observed.

The finding that enucleated patients adapt over time to their situation by generating proportionally larger lateral and vertical head movements supports the conclusions made by other research groups 3,4 that a training programme for recently enucleated patients should be initiated to teach the value of producing these movements when performing depth judgements, especially in the execution of skilled motor tasks.

This research was supported by the Natural Sciences and Engineering Research Council of Canada grant no. A6313 to M.A.G. We would like to thank Dr L. H. Allen for his assistance in acquiring patients and J. Orphan and D. Pulham for their technical expertise in the construction of the head-piece and maintenance of the WATSMART system. Last but not least, we would like to thank the patients for the cooperation and good humour that they showed throughout testing.

Key words: Distance estimation, Enucleation, Human, Limb movements, Prehension, Visuomotor behaviour.

\section{REFERENCES}

1. Allara RD, Linberg JV, Tillman W, Odom JV. Visual function in monocular patients following enucleation. Invest Ophthalmol Vis Sci 1986;27:106.

2. Schwartz TL, Linberg JV, Tillman W, Odom JV. Monocular depth and vernier acuities: a comparison of binocular and uniocular subjects. Invest Ophthalmol Vis Sci 1987;28:304.

3. Gonzalez EG, Steinbach MJ, Ono H, Wolf ME. Depth perception in children enucleated at an early age. Clin Vis Sci 1989;4:173-7.

4. Steinbach MJ, Ono $\mathrm{H}$, Wolf ME. Motion parallax judgements of depth as a function of the direction and type of head movement. Can J Psychol 1991;45:92-8.

5. Marotta JJ, Perrot TS, Nicolle D, Servos P, Goodale MA. Adapting to monocular vision: grasping with one eye. Assoc Res Vis Ophthalmol Sci Annu Meeting Abstr Issue 1994;35:2030.

6. Lappin JS. Perceiving environmental structure from optical motion. In: Johnson WW, Kaiser MK, editors. Visually guided control of movement. Moffett Field, CA: Ames Research Centre; 1991:39-60.

7. Simpson WA. Optic flow and depth perception. Spatial Vis ??;7:35-75.

8. Oldfield RC. The assessment and analysis of handedness: the Edinburgh inventory. Neuropsychologia 1971;9:97-112. 